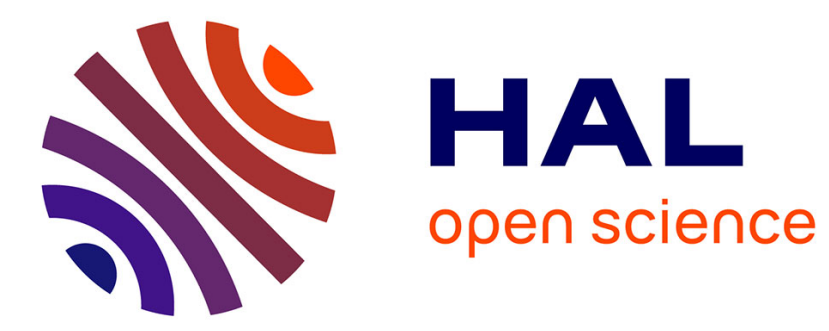

\title{
High resolution optical spectroscopy in neon using a tunable laser and an excited atomic beam
}

\author{
R.-J. Champeau, J.-C. Keller
}

\section{To cite this version:}

R.-J. Champeau, J.-C. Keller. High resolution optical spectroscopy in neon using a tunable laser and an excited atomic beam. Journal de Physique Lettres, 1975, 36 (6), pp.161-164. 10.1051/jphyslet:01975003606016100 . jpa-00231178

\section{HAL Id: jpa-00231178 https://hal.science/jpa-00231178}

Submitted on 1 Jan 1975

HAL is a multi-disciplinary open access archive for the deposit and dissemination of scientific research documents, whether they are published or not. The documents may come from teaching and research institutions in France or abroad, or from public or private research centers.
L'archive ouverte pluridisciplinaire HAL, est destinée au dépôt et à la diffusion de documents scientifiques de niveau recherche, publiés ou non, émanant des établissements d'enseignement et de recherche français ou étrangers, des laboratoires publics ou privés. 


\title{
HIGH RESOLUTION OPTICAL SPECTROSCOPY IN NEON USING A TUNABLE LASER AND AN EXCITED ATOMIC BEAM
}

\author{
R.-J. CHAMPEAU and J.-C. KELLER
}

\author{
Laboratoire Aimé-Cotton, C.N.R.S. II, Bât. 505, 91405 Orsay, France
}

(Reçu le 10 mars 1975, accepté le 10 avril 1975)

\begin{abstract}
Résumé. - La structure hyperfine de trois raies d'absorption du néon a été étudiée à l'aide d'un laser à colorant monomode et accordable et d'un jet atomique excité par bombardement électronique. Des valeurs préliminaires sont données pour les constantes $A$ et $B$ des niveaux $2 \mathrm{p}_{2}, 2 \mathrm{p}_{4}$ et $2 \mathrm{p}_{6}$ (unité $: 10^{-3} \mathrm{~cm}^{-1}$ ) :

$$
\begin{array}{lll}
A\left(2 \mathrm{p}_{2}\right)=2,45 \pm 0,15, & A\left(2 \mathrm{p}_{4}\right)=-10,43 \pm 0,15, & A\left(2 \mathrm{p}_{6}\right)=-6,19 \pm 0,15, \\
B\left(2 \mathrm{p}_{2}\right)=0,16 \pm 0,20, & B\left(2 \mathrm{p}_{4}\right)=1,51 \pm 0,20, & B\left(2 \mathrm{p}_{6}\right)=-2,20 \pm 0,20 .
\end{array}
$$
\end{abstract}

Abstract. - The hyperfine structures of three absorption lines of neon have been investigated using a single mode tunable dye laser illuminating an atomic beam excited by electron bombardment. Preliminary values for the $A$ and $B$ factors of the $2 \mathrm{p}_{2}, 2 \mathrm{p}_{4}$ and $2 \mathrm{p}_{6}$ levels are given (in $10^{-3} \mathrm{~cm}^{-1}$ ) :

$$
\begin{array}{lll}
A\left(2 \mathrm{p}_{2}\right)=2.45 \pm 0.15, & A\left(2 \mathrm{p}_{4}\right)=-10.43 \pm 0.15, & A\left(2 \mathrm{p}_{6}\right)=-6.19 \pm 0.15 \\
B\left(2 \mathrm{p}_{2}\right)=0.16 \pm 0.20, & B\left(2 \mathrm{p}_{4}\right)=\quad 1.51 \pm 0.20, & B\left(2 \mathrm{p}_{6}\right)=-2.20 \pm 0.20
\end{array}
$$

1. Introduction. - In the past few years, the use of tunable single-mode lasers in conjunction with atomic beams for high resolution optical spectroscopy has proved to be a powerful method. Up to now this method was restricted to atoms for which a direct or a stepwise excitation from the ground state is possible by means of tunable lasers (see e.g. [1]). When the resonance wavelengths are not in the proper spectral range (i.e. visible or near U.V.) the method cannot be applied. Neon and other noble gases are examples of this situation.

On the other hand, precise measurements of the hyperfine structure of the levels of neon are only available in few cases. This precludes a deep theoretical analysis of the hyperfine structure problem, taking into account the influence of relativistic corrections, far configuration mixing, etc.... Due to the complexity and relative smallness of the structures, conventional spectroscopy, even in its more recent refinements, does not allow any progress in this domain. It is therefore useful to extend the scope of the new spectroscopic techniques using tunable lasers to atoms which are in an excited level. For this, we have used an atomic beam of neon excited by an electron gun and we have performed an absorption experiment from one of the metastable levels populated by electron impact. The level scheme of the different transitions involved and the principle of the experiment are briefly discussed in section 2 of the paper, the different parts of the experimental set-up are described in section 3 and the results obtained are given in section 4 .

2. Principle of the absorption experiment. - The lower part of the energy diagram of neon is shown in figure 1. The fundamental level is $2 p^{6}\left({ }^{1} S_{0}\right)$; the lowest excited configuration $2 \mathrm{p}^{5} 3 \mathrm{~s}$ contains two metastable levels $\left({ }^{3} \mathrm{P}_{0}\right.$ and $\left.{ }^{3} \mathrm{P}_{2}\right)$ and is connected to the higher $2 p^{5} 3 p$ configuration by strong visible lines. The wavelengths of the resonance lines are below $750 \AA$ so that an optical excitation from the fundamental level to populate the excited levels would be very difficult to carry out experimentally. However the long lived metastable levels can be populated for instance by electronic bombardment, so that an absorption experiment using a dye laser can be performed.

When the laser frequency is resonant with a given component of the absorption line of neon (transition $2 p^{5} 3 s \rightarrow 2 p^{5} 3 p$ ), an increase of the population of the upper level of the transition takes place and, as a consequence, the intensity of the fluorescence light emitted from this level increases (the transition scheme of the experiment is illustrated in figure 1 for a particular pair of laser and fluorescence wavelengths). 


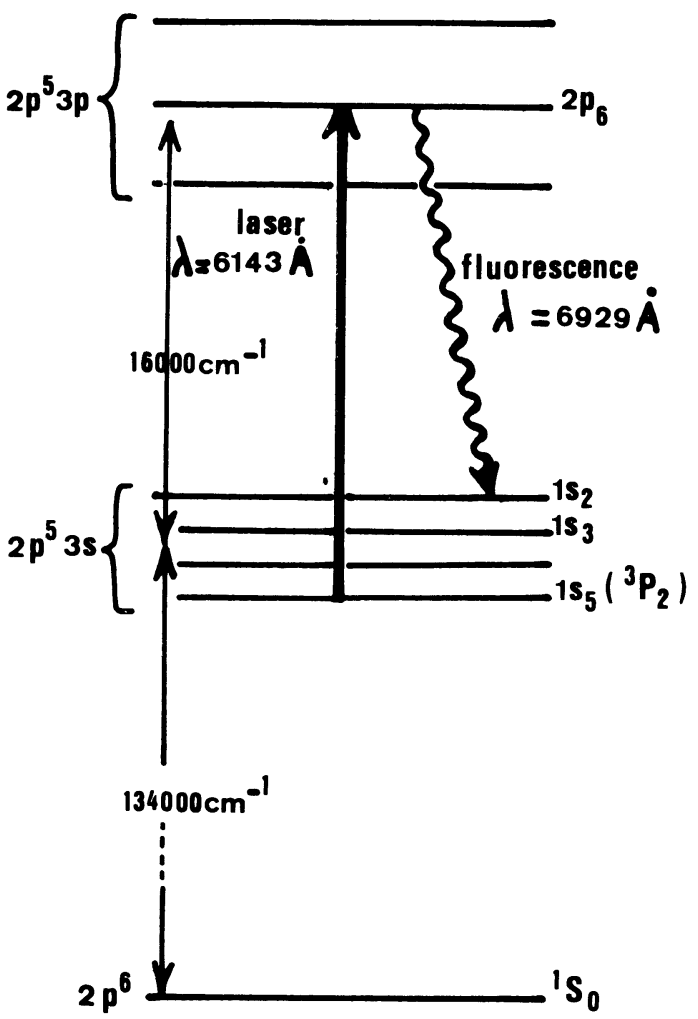

FIG. 1. - Energy diagram of neon and levels involved in a particular absorption experiment.

As the total fluorescence flux is proportional to the population of the upper level, the experiment should provide directly the profile and the structure of the absorption line by measuring the variations of the fluorescence intensity when the laser frequency is scanned. In fact the situation is more complex : the excitation by electron impact does not populate equally the different Zeeman states of the lower level of the transition [2], and furthermore, the laser light being linearly polarized, only $\Delta M=0$ transitions are allowed (taking the direction of polarization of light as the quantization axis). Therefore the fluorescence light is not emitted isotropically. As we detect only the light emitted in a limited solid angle, we do not exactly measure the population of the upper level. For these two reasons and also due to saturation effects, the relative intensities of the components which we are recording are not the same as in an usual absorption experiment.

Let us now consider the spectroscopic problems of interest in neon. Neon $(Z=10)$ has three stable isotopes (mass number 20, 21 and 22 ) $;{ }^{21} \mathrm{Ne}$ has a nuclear spin equal to $\frac{3}{2}$. Thus the structure of the absorption line is due both to isotope shift and to hyperfine structure. As the isotope shifts $\left({ }^{22} \mathrm{Ne}-{ }^{20} \mathrm{Ne}\right)$ have been accurately measured previously for the $2 p^{5} 3 p \rightarrow 2 p^{5} 3 s(2 p \rightarrow 1 s)$ lines [3] we are mainly interested in the values of the $A$ and $B$ hyperfine structure constants of ${ }^{21} \mathrm{Ne}$ and also in the oddeven isotope shifts.
3. Experimental arrangements. - 3.1 GASEOUS ATOMIC BEAM. - The atomic beam system constructed a few years ago by R. W. Stanley in our laboratory is similar to that of Purdue University. We refer to papers [4] and [5] for the description of the beam forming system and of the electron gun.

As we use enriched isotope samples, the gas must be recirculated. In order to remove the impurities, the gas is passed through a liquid nitrogen cooled trap and then on a titanium metal wire operating at high temperature.

3.2 The tUnABLE MONOCHROMATIC RADiation SOURCE. - The C.W. single-mode dye laser used in this experiment has been perfected by S. Liberman and J. Pinard [6].

The precise calibration of the laser frequency is obtained by means of a sigmameter [7]. With this instrument, the laser frequency can be changed by increments of $7.5 \mathrm{MHz}$.

The light from the laser is passed through a low resolution monochromator to eliminate the undesirable fluorescence of the dye cell before crossing the atomic beam.

The atomic beam, the electron beam and the laser beam are perpendicular to each other, but the three beams do not cross at the same point. After leaving the last collimating slit, the atoms first cross the electron beam and, a few millimeters farther, the laser beam. This allows to separate the useful fluorescence light from the natural emission of the atomic beam taking place in the region where it crosses the electron beam, without loosing many of the metastable atoms.

3.3 THE DETECTION OF RESONANCES. - The atoms excited in the upper level (configuration $2 p^{5} 3 p$ ) can decay towards several levels of the configuration $2 \mathrm{p}^{5} 3 \mathrm{~s}$. It would seem worthwhile to collect the light emitted on all corresponding wavelengths. However, in order to eliminate, as well as possible, the light scattered in the beam chamber (laser light, light, emitted by the excited part of the atomic beam, thermal emission from the heated parts of the gun, ...), we prefer to isolate only one of the fluorescence wavelengths (as different as possible from the wavelength of the absorption line) by means of an appropriate interference filter. Furthermore, as the arrangement of the windows of the beam chamber forces us to collect the fluorescence light emitted in the same direction as the laser beam, a little screen is used to block the laser light. The fluorescence light is collected by a lens and received by a photomultiplier.

A typical recording obtained with a mixture of ${ }^{20} \mathrm{Ne}$ and ${ }^{22} \mathrm{Ne}$ is shown in figure 2.

The linewidth is about $3 \times 10^{-3} \mathrm{~cm}^{-1}$ mainly due to the low collimation ratio of the beam.

4. Hyperfine structure measurements. - We have performed measurements for three different neon 


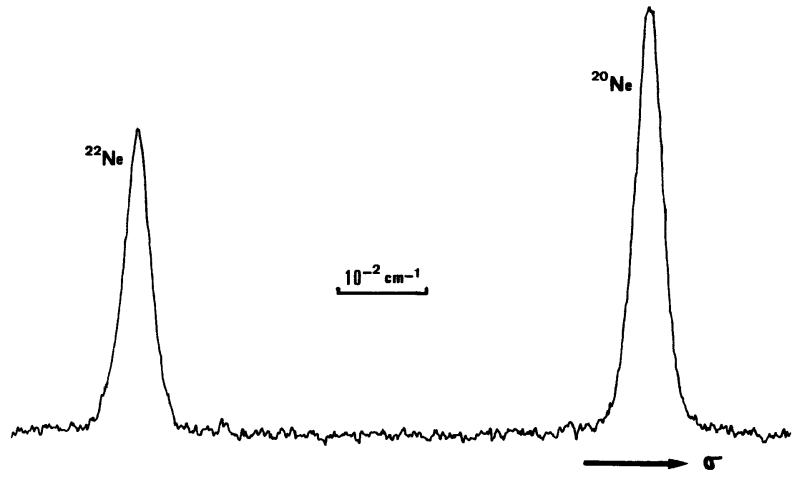

Fig. 2. - Recording for the $\lambda=5882 \AA$ line with a mixture of ${ }^{20} \mathrm{Ne}$ and ${ }^{22} \mathrm{Ne}$. The isotope shift is $\delta \sigma=57.7 \times 10^{-3} \mathrm{~cm}^{-1}$.

lines : $\lambda=5882 \AA \quad\left(2 \mathrm{p}_{2} \rightarrow 1 \mathrm{~s}_{5}\right), \lambda=5945 \AA$ $\left(2 \mathrm{p}_{4} \rightarrow 1 \mathrm{~s}_{5}\right)$ and $\lambda=6143 \AA\left(2 \mathrm{p}_{6} \rightarrow 1 \mathrm{~s}_{5}\right)$; the fluorescence lines used for detection were respectively : $\lambda=6599 \AA\left(2 \mathrm{p}_{2} \rightarrow 1 \mathrm{~s}_{2}\right), \lambda=6678 \AA\left(2 \mathrm{p}_{4} \rightarrow 1 \mathrm{~s}_{2}\right)$ and $\lambda=6929 \AA\left(2 \mathrm{p}_{6} \rightarrow 1 \mathrm{~s}_{2}\right)$.

For these three different lines we have measured once more the ${ }^{22} \mathrm{Ne}-{ }^{20} \mathrm{Ne}$ isotope shifts ; our measurements agree with those given in [3].

Using a neon sample with $91 \%{ }^{21} \mathrm{Ne}, 5.7 \%{ }^{22} \mathrm{Ne}$ and $3.3 \%{ }^{20} \mathrm{Ne}$ we have recorded the hyperfine structure of the three quoted lines (Fig. 3). The structure

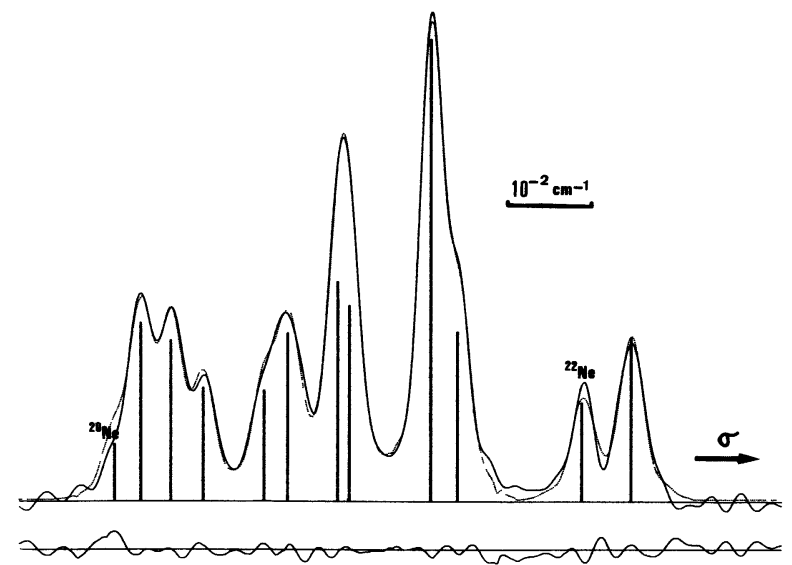

Fig. 3. - Hyperfine structure of the line $\lambda=6143 \AA$. The experimental recording has been mathematically filtered and then fitted with a calculated profile. The lower trace shows the difference between the experimental and calculated curves. of the lines is only partially resolved and a theoretical fit is necessary to locate the position of the components. The $A$ and $B$ values for the lower level of the transitions $\left(1 \mathrm{~s}_{5}\right)$ are known from the very precise experiment of [8] and the even-even isotope shifts are also precisely known [3]. So the relative positions of the components only depend on the $A$ and $B$ values of the upper level of the transition and on the oddeven isotope shift. As, in the h.f.s. recordings, no component is sufficiently well isolated, the profile has to be fitted numerically to the experimental data as well as the position parameters. To do this we use a computer code of O. Robaux [9], following a procedure already described (see e.g. [10]). Unfortunately as already said (cf. 2) the recorded intensity pattern is not the one that can be calculated using the isotopic abundances and the relative hyperfine intensities in a multiplet. Then the intensities must be considered as free parameters in the fitting procedure. It was verified that the values obtained for the $A$ and $B$ parameters are almost insensitive to the values of the intensities as far as these values remain reasonable although the recorded profile reconstruction is very sensitive to them.

The hyperfine structure results are given in table I; they are compared with other measurements ([10-14]) and with theoretical values [15]. The values given in table $I$ are average values of the various recordings of the same line; to have an indication of the precision, the standard deviations have been calculated for each set of recordings but, as the number of recordings is too small, we have attributed to the three $A$ values, the same uncertainty equal to twice the average of the corresponding standard deviations. The uncertainties of the $B$ factor have been evaluated in the same way.

The $A$ and $B$ values are in rather good agreement with the theoretical predictions of S. Liberman [15]. However, small discrepancies between measured and calculated $A$ values must be noticed; a more detailed theoretical analysis of the magnetic hyperfine structure will therefore probably be possible using the $A$ values obtained in the recent experiments (This work and ref. [14]). For the $B$ factors, the precision is yet unsufficient to show any discrepancy between theory and experiment.

TABLE I

Hyperfine structure constants of ${ }^{21} \mathrm{Ne}\left(\right.$ in $\left.10^{-3} \mathrm{~cm}^{-1}\right)$.

$\begin{array}{ccccccc} & A\left(2 \mathrm{p}_{2}\right) & B\left(2 \mathrm{p}_{2}\right) & A\left(2 \mathrm{p}_{4}\right) & B\left(2 \mathrm{p}_{4}\right) & A\left(2 \mathrm{p}_{6}\right) & B\left(2 \mathrm{p}_{6}\right) \\ \text { This work } & 2.45 \pm 0.15 & 0.16 \pm 0.20 & -10.43 \pm 0.15 & 1.51 \pm 0.20 & -6.19 \pm 0.15 & -2.20 \pm 0.20 \\ {[10]} & & & -10.4 \pm 0.4 & 1.7 \pm 1.1 & & \\ {[11]} & & & -10.4 \pm 0.3 & |B|<2 & \\ {[14]} & 2.51 \pm 0.03 & & -10.32 \pm 0.04 & 1.64 \pm 0.02 & -6.19 \pm 0.03 & -2.29 \pm 0.02 \\ {[12]} & 2.5 & (0) & -7.3 \pm 0.1 & & & \\ {[13]} & & & -10.06 & 1.60 & -6.11 & -2.31 \\ {[15]} & 2.83 & 0.015 & -10 & & & \end{array}$


The values for the ${ }^{22} \mathrm{Ne}-{ }^{21} \mathrm{Ne}$ isotope shifts of the lines $\lambda=6143 \AA, 5945 \AA$ and $5882 \AA$ were found to be respectively : (in $10^{-3} \mathrm{~cm}^{-1}$ ) : 26.4, 27.4 and 28.6. Although these values are in fairly good agreement with predictions deduced from the mass shift measurements of the ${ }^{21} \mathrm{Ne}-{ }^{22} \mathrm{Ne}$ shift : indeed, the low concentration of the even isotopes in the ${ }^{21} \mathrm{Ne}$ sample does not allow an accurate determination of their positions.

5. Conclusion. - The hyperfine structure measurements given in table I are only preliminary results and a greater accuracy should be possible in the near future, for example by decreasing the residual Doppler- width. This, and other technical improvements are under way.

However we have shown in the case of neon that laser high resolution spectroscopy with atomic beams can be applied to atoms with resonant wavelengths out of the spectral range of tunable lasers and for which an absorption experiment from a metastable state is possible.

Acknowledgments. - The authors would like to thank P. Juncar and J. Pinard for their help in the use of the sigmameter and of the dye laser, and Pr. R. W. Stanley for benevolent advices and informations concerning the atomic beam apparatus.

\section{References}

[1] Duong, H. T. and Vialle, J.-L., Opt. Commun. 12 (1974) 71 ; Hartig, W. and Walther, H., Appl. Phys. 1 (1973) 171; LANGe, W., Luther, J., NotTBeCK, B. and Schroeder, H. W., Opt. Commun. 8 (1973) 157.

[2] Mott, N. F. and Massey, H. S. W., Theory of Atomic Collisions (Clarendon Press, Oxford), 1950

[3] Odintsov, V. R., Opt. Spectros. 28 (1965) 205.

[4] Stanley, R. W., J. Opt. Soc. Am. 56 (1966) 350.

[5] Larson, H. P., Thesis (1967) Purdue University.

[6] Liberman, S. and Pinard, J., Opt. Commun. 24 (1974) 142.

[7] JunCaR, P. and PinaRd, J., to be published.
[8] Grosof, G. M., Buck, P., Lichten, W. and Rabi, I. I., Phys. Rev. Lett. 1 (1958) 214.

[9] Robaux, O., Opt. Acta 18 (1971) 523.

[10] Champeau, R.-J., Keller, J.-C., Robaux, O. and Vergès, J., J. Phys. B 7 (1974) L-163.

[11] Champeau, R.-J. and Keller, J.-C., J. Phys. B 6 (1973) L-76.

[12] Rasmussen, F. and Middelboe, V., Mat. fis. 32 (1960) 1.

[13] Ducas, T. W., Feld, M. S., Ryan, L. W., Skribanowitz, N. and Javan, A., Phys. Rev. 5 (1972) 1036.

[14] Giacobino, E., J. Physique Lett. 36 (1975) L-65.

[15] Liberman, S., Physica 69 (1973) 598. 